\title{
Obstacle Avoidance of a Class of Underactuated Robot Manipulators: GA based approach
}

\author{
Qingbo LIU, Yueqing YU, Shanzeng LIU \\ College of Mechanical Engineering \& Applied Electronics Technology, Beijing University of Technology \\ Beijing, 100124, China \\ E-mail:lqb@emails.bjut.edu.cn \\ Milan ZHANG \\ College of Mechanical Electronic and Control Engineering, Beijing Jiao tong University, \\ Beijing, 100044, china \\ E-mail:lqb0123@gmail.com \\ www.bjut.edu.cn
}

Received: 21-05-2008

Revised: $06-10-2008$

\begin{abstract}
This paper presents a practical collision-free motion planning method for general underactuated robot manipulators. First the dynamic properties of underactuated robot manipulator are analyzed, and then the collision avoidance problem is formulated and solved as a position-based force control problem. Virtual generalized force representing the intrusion of the arm into the obstacle dangerous zone is computed in real time using a virtual spring-damper model. The partly stable controllers are adopted and the energy based fitness function is built, then the best switching sequence of partly stable controllers is obtained by genetic algorithm. Because the proposed method does not make any hypothesis about the degree of freedom, it can be used without modification for arms with a large number of degree of freedom. At last numerical simulations are carried out to illuminate the validity of the proposed method.
\end{abstract}

Keywords: underactuated robots, obstacle avoidance, motion planning, genetic algorithm

\section{Introduction}

Underactuated robots are those which have fewer control inputs than the degrees of freedom of the system. As far as the underactuated manipulators are concerned, they have one or more joints without actuators, namely these joints are passive or free. Hyper-redundant (snakelike) robots with passive joints, legged robots with passive joints, gymnastic robots (Acrobot), fault tolerance robot systems, undersea vehicles and mobile robots are some examples of the underactuated systems ${ }^{1}$, 2. However, control of such kind of system is a complicated task because of the intrinsic characteristics such as complex nonlinear dynamic, nonholonomic behavior and lack of linearizability exhibited in this kind of nonlinear systems ${ }^{3}$. It is necessary to explore new control techniques to overcome the problem.

An extensive amount of research on the kinematics and dynamics of robots has been carried out for regular (full actuated) manipulators. There is an independent generalized force for each degree of freedom that can be applied by a control actuator. But for underactuated robots, the generalized coordinates are not independent and the control objective can be realized only by the dynamic coupling between the active and passive joints ${ }^{4}$. In most cases, the underactuated manipulators with two or more degree of freedoms are second-order nonholonomic systems ${ }^{5}$. That is to say that the systems have accelerations-dependent constraints which are not 
integrable to obtain velocity or configuration dependent constraints.

Collision-free motion planning of manipulators is a weakness of current robotic technology. Many typical methods for manipulators in collision-free motion planning are based on graph searching in finite discrete C-space ${ }^{6,7}$. Kando pointed out that describing entire Cspace representation is a waste of time when the environment frequently changes, because it is not always necessary prior to path searching ${ }^{6}$.In his method, collision-freeness is checked only on grid points of the discrete C-space and whose arc/edges denote adjacent relations between the grid points, then a graph search method call bi-direction heuristic front-to-front algorithm is applied to find paths. Lozano proposed slice projection method to describe the entire C-space ${ }^{8}$, it is practically difficult to compute slice projection of manipulators with more the 4 degree of freedoms. Although this method is more effective than other methods needed for the entire resolution of the discrete C-space representation, its computational cost increases exponentially as dof resolution of the discrete C-space increases. Kevin proposed a method for collision-free trajectory planning of a 3-dof robot with a passive joint ${ }^{9}$, the problem of planning feasible trajectories in the robot's six-dimensional state space are decoupled into the computationally simpler problems of planning path in the three-dimensional configuration space and time scaling the paths according to the manipulator dynamics. Shingo $^{10}$ proposed a practical path planning method than can find collision-free paths quickly for a general robot manipulator. In his method global search tries to find a sequence of intermediate goals and serial local search tried to sequentially find local paths between each two adjacent sub-goals.

Even though many researchers have carried out considerable numbers of studies in collision-free trajectory planning for full actuated systems; there are few researches for underactuated robots. From this point of view, this paper presents a universal method for collision avoidance of underactuated robot manipulators. The rest of the paper is organized as follows: in section 2 the dynamic model of underactuated robots is introduced, the collision-free trajectory planners are discussed in section 3 , best switching sequence searching of partly stable controllers are discussed in section 4 and numerical simulations are carried out in section5, finally some discussions and conclusions are made in section 6 .

\section{Dynamic Equation}

Consider the dynamics of an $n$-degree-of-freedom mechanical system with $m$ actuators; the kinetic energy of the manipulator is defined as:

$$
K=\frac{1}{2} \sum_{j=1}^{n} \sum_{i=1}^{n} m_{i j}(q) \dot{q}_{i} \dot{q}_{j}=\frac{1}{2} \dot{q}^{T} M(q) \dot{q}
$$

Where $q \in \mathfrak{R}^{n}$ and $\dot{q} \in \mathfrak{R}^{n}$ are the vectors of joint positions and joint velocities of the manipulator, respectively, and $M(q) \in \mathfrak{R}^{n \times n}$ is the inertia matrix of the manipulator. The matrix $M(q)$ is symmetric and positive definite for each $q \in \mathfrak{R}^{n}$.The Lagrange function $L$ of the system is given by:

$$
L=K-V
$$

Where $V$ is the potential energy. The Lagrange equation is defined as follows

$$
\frac{d}{d t}\left(\frac{\partial L}{\partial \dot{q}}\right)-\frac{\partial L}{\partial q}=\tau
$$

Where $\tau \in R^{n \times 1}$ are generalized forces. The dynamic equations in matrix-vector form are given by:

$$
M(q) \ddot{q}+C(q, \dot{q}) \dot{q}=\tau
$$

Where $C(q, \dot{q}) \dot{q}$ represent the element of Coriolis, Centrifugal and viscous friction vector. Decompose the active and passive variables from (4) we can get

$$
\begin{gathered}
M_{a a} \ddot{q}_{a}+M_{a p} \ddot{q}_{p}+h_{a}=\tau_{a} \\
M_{p a} \ddot{q}_{a}+M_{p p} \ddot{q}_{p}+h_{p}=0
\end{gathered}
$$

Where " $a$ " stand for active joint," $p$ " are the passive joints variables, $\tau_{\mathrm{a}}$ are the active joint torques, $h$ is the element of Coriolis, Centrifugal and viscous friction vector and with the expression as follows

$$
h=M(q) \dot{q}-\frac{1}{2} \dot{q}^{T} \frac{\partial}{\partial q}(M(q)) \dot{q}
$$

Equation (5b) represents a dynamic constraint on the system. Usually the necessary and sufficient condition for the partial integrability for the constraint is not satisfied. Hence the system is often second-order nonholonomic system. From (5b) we may solve for $\ddot{q}_{p}$ as

$$
\ddot{q}_{p}=-M_{p p}^{-1}(q)\left[M_{p a}(q) \ddot{q}_{a}+h_{p}\right]
$$

We choose $\ddot{q}_{a}$ as control input, (5b) can be rewritten as

$$
\left\{\begin{array}{l}
\ddot{q}_{a}=u \\
\ddot{q}_{p}=J(q) \ddot{q}_{a}+R(q, \dot{q})
\end{array}\right.
$$


Where

$$
\begin{aligned}
& J(q)=-M_{p p}^{-1}(q) M_{p a}(q) \\
& R(q, q)=-M_{p p}^{-1} h_{p}
\end{aligned}
$$

Note that if $(q, \dot{q})=\left(q_{e}, 0\right)$ is an equilibrium solution we refer to $q_{\mathrm{e}}$ as an equilibrium configuration. Equation (8) can be expressed in the usual nonlinear control system form by defining the following state variables

$$
x_{1}=q_{a}, x_{2}=q_{p}, x_{3}=\dot{q}_{a}, x_{4}=\dot{q}_{p}
$$

Then the state equations are given by

$$
\begin{aligned}
& \dot{x}_{1}=x_{3} \\
& \dot{x}_{2}=x_{4} \\
& \dot{x}_{3}=u \\
& \dot{x}_{4}=J\left(x_{1}, x_{2}\right) u+R\left(x_{1}, x_{2}, x_{3}, x_{4}\right)
\end{aligned}
$$

Equation (11) defines a drift vector field $f(\mathrm{x})=\left(x_{3}, x_{4}, 0, R\left(x_{1}, x_{2}, x_{3}, x_{4}\right)\right)$ and control vector fields $g_{\mathrm{i}}(\mathrm{x})=\left(0,0, e_{\mathrm{i}}, J_{\mathrm{i}}\left(x_{1}, x_{2}\right)\right)$, where $e_{\mathrm{i}}$ denotes the $i$ th standard basis vector in $\mathfrak{R}^{m}$ and $J_{\mathrm{i}}\left(x_{1}, x_{2}\right)$ denotes the $i$ th column of the matrix function $J\left(x_{1}, x_{2}\right), i=1, \ldots, m$, according to the standard control system form

$$
\dot{x}=f(x)+\sum_{i=1}^{m} g_{i}(x) u_{i}
$$

We first demonstrate that the second-order nonholonomic system, defined by equations (8), does satisfy certain nonlinear controllability properties ${ }^{11,12}$. In particular, we show that the system is strongly accessible.

Theorem 1: Let $n-m \geq 1$. The underactuated robot system, defined by (8) is strongly accessible.

Theorem 2:Assume that $R_{\mathrm{i}}(q, 0)=0, \forall q \in Q$, for some $i \in\{1, \cdots, n-m\}$, let $n-m \geq 1$ and let $\left(q^{e}, 0\right)$ denote an equilibrium solution. Then the underactuated system, defined by (8) is not asymptotically stabilized to $\left(q^{e}, 0\right)$ using time-invariant continuous (static or dynamic) state feedback law.

Theorem 3: Let $n-m \geq 1$ and let $\left(q^{e}, 0\right)$ denote an equilibrium solution, the underactuated system, defined by (8) is small time locally controllable at $\left(q^{e}, 0\right)$ if there exists a set of $n-m$ pairs of indexes $\left(i_{k}, j_{k}\right) \in I_{m}^{2}, i_{k} \neq j_{k}, k \in I_{n-m}$,such that

$$
\operatorname{dimspan}\left\{H_{i_{k} j_{k}}\left(q^{e}\right), k \in I_{n-m}\right\}=n-m
$$

and

$$
H_{i_{k} j_{k}}\left(q^{e}\right)=0, \forall k \in I_{n-m}
$$

Accessibility and controllability analysis are very important and necessary for the control of underactuated robot manipulators. In the next section we mainly describe the obstacle avoidance strategy of underactuated robot manipulators.

\section{Collision Free Trajectory Planning}

Robotic manipulators are basically positioning devices that can carry payloads by their end-effectors from initial positions and orientations to target destinations along prescribed Cartesian paths. A robotic system must have the ability to cope with complex environments, including when there are obstacles in workspace. In this paper collision avoidance is accomplished with the help of modification of the generalized forces which are generated by the virtual spring-damper model.

\subsection{Virtual Spring-damper Model}

For every reachable object in the manipulator workspace, we define a dangerous zone displaced from the object surface by a user-specified stand-off distance. Inside the dangerous zone, there are fictitious springs with natural length and user-defined constant stiffness coefficient, in parallel with dampers with user-specified constant damping coefficient $k_{\mathrm{p}}$ occupying the space between the object surface and the dangerous zone boundary, a typical example for underactuated manipulators is shown in Fig. 1.

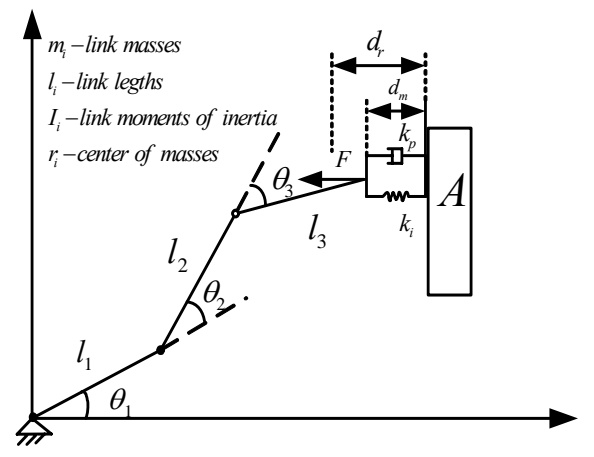

Fig. 1 Planar $3 R R \bar{R}$ underactuated manipulator

When the obstacle detection system does not detect intrusion into any dangerous zones $\left(d_{m} \geq d_{r}\right)$, the userspecified nominal path is executed and the arm performs an obstacle-free motion. As soon as any point on the arm enters the dangerous zone which is determined by the obstacle detection system $\left(d_{m}<d_{r}\right)$, a virtual intrusion force is generated and is exerted on the arm at the intrusion point (see Fig. 1,A is the obstacle) to cause deviation from the nominal path. The magnitude of this force is related directly to the extent and the rate of 
intrusion into the dangerous zone, the intrusion force $F$ can be computed as:

$$
F=k_{i} e+k_{p} \frac{d e}{d t}
$$

Where $k_{i}$ is the stiffness coefficient, $k_{p}$ is the damping coefficient, $e$ is the compression deformation of the virtual spring and with the expression of $e=d_{r}-d_{m}, k_{i} e$ represents the compressive force due to the spring, while the term $k_{p}(d e / d t)$ is the resistive force due to the damper. When the manipulators are in the safety zone the compression deformation equals to zero, so we can get the expression of $e$ in the whole workspace:

$$
e= \begin{cases}0, & d_{m} \geq d_{r} \\ d_{r}-d_{m}, & d_{m}<d_{r}\end{cases}
$$

From Fig.2 it can be shown that the virtual intrusion force $F$ is always along the line of shortest length $P Q$ connecting the closest points $P$ on the obstacle and on the $\operatorname{arm} Q$, where $d_{m}=|P Q|$.

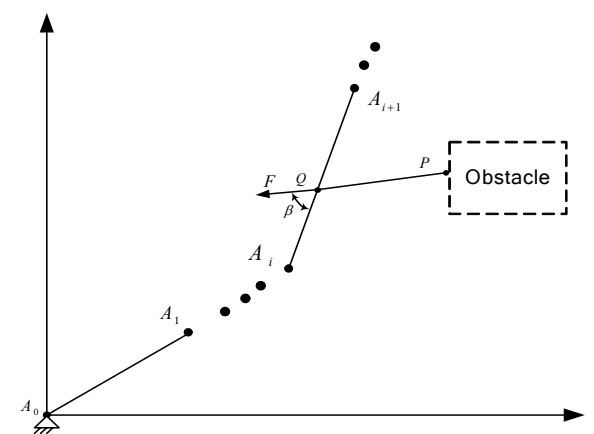

Fig. 2 Local schematic diagram between obstacle and robot manipulators

\subsection{Modification of Generalized Forces}

We can get the value of the virtual intrusion force by (16).Considering the situation when the arms interfere with dangerous zone as shown in Fig.2, then the nonholonomic constraints equations of the dynamic model can be modified as follows

$$
M_{p a}{ }^{i+1} \ddot{\theta}_{a}^{i+1}+M_{p p}{ }^{i+1} \ddot{\theta}_{p}{ }^{i+1}+h_{p}^{i+1}=\tau_{v}
$$

Where $i+1$ stands for the intrusion force $F$ acting on the passive link $A_{i} A_{i+1}, \tau_{v}$ is the virtual passive torques generated by the intrusion force and has the expression:

$$
\tau_{v}=F \times\left|A_{i} Q\right| \times \sin (\beta)
$$

Where $\beta$ is the included angel between link $A_{i} A_{i+1}$ and $P Q$. If $A_{i} A_{i+1}$ is active link, the intrusion force $F$ will be acted on the nearest passive joint of $A_{i}$ and the equivalent virtual torque on joint $A_{i}$ can easily be gotten. Equation (17) is the basis of collision-free motion planning for underactuated manipulators.

\subsection{Partly Stable Controllers}

The objective of motion planning is to find a feasible motion from the initial state to the desire state. Here partly stable controllers are adopted ${ }^{13}$. For each link we can design partly stable controllers as:

$$
\ddot{q}_{i}=\ddot{q}_{i d}+k_{v i}\left(\dot{q}_{i d}-\dot{q}\right)+k_{p i}\left(q_{i d}-q_{i}\right)
$$

Where $q_{i d}, \dot{q}_{i d}$ and $\ddot{q}_{i d}(i=1 \cdots n)$ are the desired joint angel positions, joint angular velocities and joint angular accelerations respectively. $k_{\mathrm{vi}}>0, k_{\mathrm{pi}>0}$ are the derivative and position gain coefficients. For $n$ degree of freedom underactuated mechanical system with $m$ actuators, $\mathrm{C}_{n}{ }^{\mathrm{m}}$ partly stable controllers can be designed totally. Define the error $e_{i}=q_{i d}-q_{i}$ and $\dot{e}_{i}=\dot{q}_{i d}-\dot{q}_{i}$. Error function of controlled coordinate for each case can be gotten:

$$
\ddot{e}_{i}+k_{v i} \dot{e}_{i}+k_{p i} e_{i}=0
$$

It can be seen from (20) that each control law guarantee the control variables converge to the desired value in finite time if the coefficients $k_{\mathrm{vi}}$ and $k_{\mathrm{pi}}$ are chosen properly. The control objective is to move the robot system to the desired configuration, and can be achieved by the proper switching of the control laws. This process is based on the evolutionary computation for searching the best combination of partly stable controllers from a set of elemental controllers.

\section{Best Switching Sequence Searching Using GA Method}

Genetic Algorithms ${ }^{14}$ are adaptive heuristic search algorithm premised on the evolutionary ideas of natural selection and genetic. The basic concept of GA is designed to simulate processes in natural system necessary for evolution, specifically those that follow the principles of survival of the fittest first laid down by Charles Darwin. As such they represent an intelligent exploitation of a random search within a defined search space to solve a problem. 


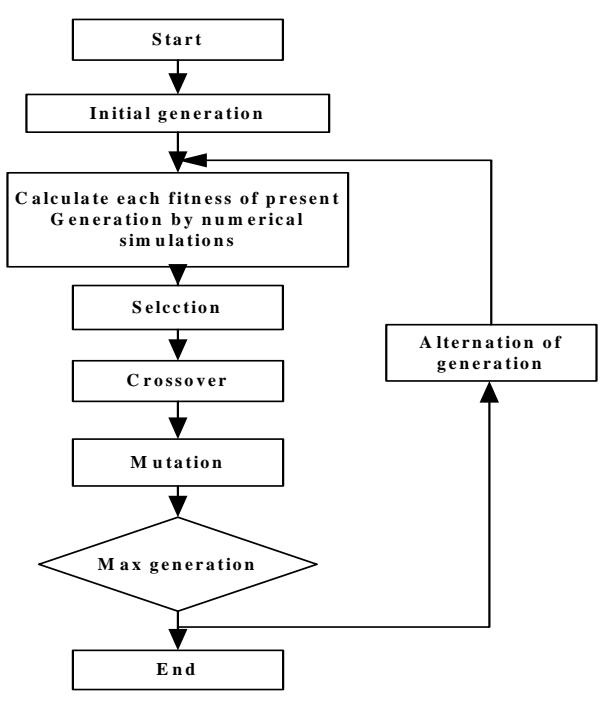

Fig. 3 GA process

Fig.3 describes the GA process when it is used. Whereas Genetic Algorithms include a variety of operators (i.e. selection, crossover and mutation), the basics of Genetic Algorithm can be described as follows: Given some initial population and the terminal generation, proceed as follows:

(1) Sort the population from the best to worst according to given cost function;

(2) Selection rules: select the individuals, called parents that contribute to the population at the next generation;

(3) Crossover rules: combine two parents to form children for the next generation;

(4) Mutation rules; apply random changes to individual parents to form children;

(5) Terminal condition judgment: if satisfied the algorithm terminated, otherwise return to (1).

The fitness function is the driving force behind the GA. The evaluation function is called from the GA to determine the fitness of each solution string generated during the search. In this paper, when there are no obstacles in workspace, the fitness function is defined as follows:

$$
\min \quad E=\sum_{i=1}^{N} e_{i} w e_{i}^{T}
$$

Where $e=\left[q_{1}-q_{1 d}, \cdots q_{n}-q_{n d} \dot{q}_{1}-\dot{q}_{1 d}, \cdots \dot{q}_{n}-\dot{q}_{n d}\right]^{T}, N$ is the final discrete time instant. Matrix $w=\operatorname{diag}\left[w_{1}, w_{2}, \cdots w_{2 n}\right]$ denotes the weights of controlled $\operatorname{error}\left(w_{1}, w_{2} \cdots w_{2 n}>0\right)$.

\section{Underactuated Robot Manipulator Example}

\subsection{Controllability and Stabilizability Analysis}

Consider a planar $3 R R \bar{R}$ (the last joint is free, i.e., with no actuator) robot as shown in Fig.1, the robot with three revolute joints, moving on a horizontal plane so that gravity can be ignored. Define the following constants

$$
\begin{aligned}
& a_{1}=m_{1} r_{1}^{2}+m_{2} l_{1}^{2}+m_{3} l_{1}^{2}+I_{1} \\
& a_{2}=m_{2} r_{2}^{2}+m_{3} l_{2}^{2}+I_{2} \\
& a_{3}=m_{3} r_{3}^{2}+I_{3} ; b_{1}=m_{2} l_{1} r_{2} ; b_{2}=m_{3} l_{1} l_{2} \\
& b_{3}=m_{3} l_{2} r_{3} ; b_{4}=m_{3} l_{1} r_{3}
\end{aligned}
$$

From part 2 the dynamic equation of $3 \mathrm{R}$ underactuated robot can written as

$$
\left[\begin{array}{lll}
m_{11} & m_{12} & m_{13} \\
m_{21} & m_{22} & m_{23} \\
m_{31} & m_{32} & m_{33}
\end{array}\right]\left[\begin{array}{l}
\ddot{q}_{1} \\
\ddot{q}_{2} \\
\ddot{q}_{3}
\end{array}\right]+\left[\begin{array}{l}
h_{1} \\
h_{2} \\
h_{3}
\end{array}\right]=\left[\begin{array}{l}
\tau_{1} \\
\tau_{2} \\
0
\end{array}\right]
$$

Where

$$
\begin{aligned}
& m_{11}= a_{1}+a_{2}+a_{3}+2 b_{1} \cos \left(q_{2}\right)+2 b_{2} \cos \left(q_{2}\right) \\
&+2 b_{3} \cos \left(q_{3}\right)+2 b_{4} \cos \left(q_{2}+q_{3}\right) \\
& m_{12}= a_{2}+a_{3}+\left(b_{1}+b_{2}\right) \cos \left(q_{2}\right)+2 b_{3} \cos \left(q_{3}\right) \\
&+b_{4} \cos \left(q_{2}+q_{3}\right) ; m_{21}=m_{12} \\
& m_{22}= a_{2}+a_{3}+b_{3} \cos \left(q_{3}\right) \\
& m_{13}= a_{3}+b_{3} \cos \left(q_{3}\right)+b_{4} \cos \left(q_{2}+q_{3}\right) \\
& m_{31}= m_{13} ; m_{22}=a_{2}+a_{3}+2 b_{3} \cos \left(q_{3}\right) \\
& m_{23}= a_{3}+b_{3} \cos \left(q_{3}\right) ; m_{32}=m_{23} \\
& m_{33}= a_{3} \\
& h_{1}=-b_{4}\left(\dot{q}_{2}+\dot{q}_{3}\right)\left(2 \dot{q}_{1}+\dot{q}_{2}+\dot{q}_{3}\right) \sin \left(q_{2}+q_{3}\right)- \\
&\left(2 \dot{q}_{1}+\dot{q}_{2}\right) \dot{q}_{2}\left(b_{1}+b_{2}\right) \sin (q 2)-b_{3}\left(2 \dot{q}_{1}+2 \dot{q}_{2}\right. \\
&\left.+\dot{q}_{3}\right) a_{3} \sin \left(q_{3}\right) \\
& h_{2}= \dot{q}_{1}^{2}\left\{\left(b_{1}+b_{2}\right) \sin \left(q_{2}\right)+b_{4} \sin \left(q_{2}+q_{3}\right)\right\}- \\
& 2 b_{3} \dot{q}_{1} \dot{q}_{3} \sin \left(q_{3}\right)-2 b_{3} \dot{q}_{2} \dot{q}_{3} \sin \left(q_{3}\right)-b_{3} \dot{q}_{3}^{2} \sin \left(q_{3}\right) \\
& h_{3}=\dot{q}_{1}^{2}\left(b_{3} \sin \left(q_{3}\right)+b_{4} \sin \left(q_{2}+q_{3}\right)\right) \\
&+2 \dot{q}_{1} \dot{q}_{2} b_{3} \sin \left(q_{3}\right)+b_{3} \dot{q}_{2}^{2} \sin \left(q_{3}\right)
\end{aligned}
$$

According to Theorem 1 to Theorem 3, we now can state the following results which characterize the controllability and stabilizability properties of the constrained $3 R R \bar{R}$ underactuated robot manipulator.

Proposition: let $\mathbf{M}^{e}$ denote the equilibrium manifold and let $\left(q^{e}, 0\right) \in \mathrm{M}^{e}$ denote an equilibrium solution. The following hold for the constrained manipulator dynamics described by (23) 
(1) The system is strongly accessible since the space spanned by vectors

$\operatorname{span}\left\{g_{1}, g_{2},\left[f, g_{1}\right],\left[f, g_{2}\right],\left[g_{2},\left[f, g_{1}\right],\left[f,\left[g_{2},\left[f, g_{1}\right]\right]\right]\right\}\right.$ has dimension six at any $(q, \dot{q}) \in \mathrm{M}$.

(2) The system is small time locally controllable at $\left(q^{e}, 0\right)$ since the sufficient conditions for STLC of Theorem 3 are satisfied.

(3)There exist both time-invariant piecewise analytic feedback laws and time-periodic continuous feedback laws which asymptotically stabilize $\left(q^{e}, 0\right)$.

(4) There is no time-invariant continuous feedback law which asymptotically stabilizes the close loop to $\left(q^{e}, 0\right)$.

\subsection{Numerical Simulation}

The virtual spring-damper method (VSDM) is compared with switching computed torque method (CTM) which was proposed by L.Udawatta ${ }^{15}$ to show the advantage of the propose method.

To solve the general problem with optimum switching of available PSCs, first define the total time span $T$. The genes of a chromosome are represented as controller indexes. For $3 R R \bar{R}$ underactuated robot, 3 PSCs can be obtained:

Control law 1:

$$
\left[\begin{array}{l}
\ddot{q}_{1} \\
\ddot{q}_{2} \\
\ddot{q}_{3}
\end{array}\right]=\left[\begin{array}{l}
\ddot{q}_{1 d}+k_{v 1}\left(\dot{q}_{1 d}-\dot{q}_{1}\right)+k_{p 1}\left(q_{1 d}-q_{1}\right) \\
\ddot{q}_{2 d}+k_{v 2}\left(\dot{q}_{2 d}-\dot{q}_{2}\right)+k_{p 2}\left(q_{2 d}-q_{2}\right) \\
-m_{33}{ }^{-1}\left(\tau_{v}+h_{3}+m_{31} \ddot{q}_{1}+m_{32} \ddot{q}_{2}\right)
\end{array}\right]
$$

Control law 2:

$$
\left[\begin{array}{l}
\ddot{q}_{1} \\
\ddot{q}_{3} \\
\ddot{q}_{2}
\end{array}\right]=\left[\begin{array}{l}
\ddot{q}_{1 d}+k_{v 1}\left(\dot{q}_{1 d}-\dot{q}_{1}\right)+k_{p 1}\left(q_{1 d}-q_{1}\right) \\
\ddot{q}_{3 d}+k_{v 3}\left(\dot{q}_{3 d}-\dot{q}_{3}\right)+k_{p 3}\left(q_{3 d}-q_{3}\right) \\
-m_{32}{ }^{-1}\left(\tau_{v}+h_{3}+m_{31} \ddot{q}_{1}+m_{33} \ddot{q}_{3}\right)
\end{array}\right]
$$

Control law 3:

$$
\left[\begin{array}{l}
\ddot{q}_{2} \\
\ddot{q}_{3} \\
\ddot{q}_{1}
\end{array}\right]=\left[\begin{array}{l}
\ddot{q}_{2 d}+k_{v 2}\left(\dot{q}_{2 d}-\dot{q}_{2}\right)+k_{p 2}\left(q_{2 d}-q_{2}\right) \\
\ddot{q}_{3 d}+k_{v 3}\left(\dot{q}_{3 d}-\dot{q}_{3}\right)+k_{p 3}\left(q_{3 d}-q_{3}\right) \\
-m_{31}{ }^{-1}\left(\tau_{v}+h_{3}+m_{32} \ddot{q}_{2}+m_{33} \ddot{q}_{3}\right)
\end{array}\right]
$$

Control law 1 to control law 3 can be expressed as the bit string code 1, 2 and 3 . So each individual can be coded as shown in Fig.4. Then the initial population is generated by a random set of $M$ "individual", here $M$ is the population size.

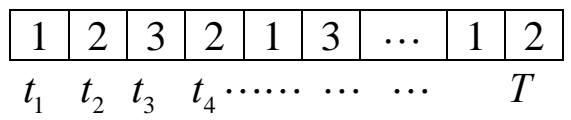

Fig.4 Coding of genes with control indices
The dynamic parameters of the planar $3 R R \bar{R}$ underactuated robot in Fig.1 are listed in Tab.1. The size of a population is 100 . The maximum number of generations is 300 . The obstacle is a circle which centered at $(0.5,0.55)$ with a radius of $0.06 \mathrm{~m}$. The gains

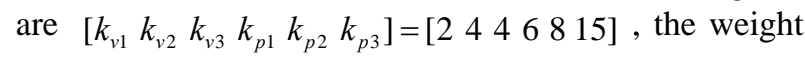
matrix $\quad w=\operatorname{diag}\left(\left[\begin{array}{llllll}10^{4} & 10^{4} & 10^{4} & 100 & 100 & 100\end{array}\right]\right) \quad$ and

\begin{tabular}{|c|c|}
\hline Conditions & Setting values \\
\hline Simulation time & $10[s]$ \\
\hline Sample interval & $0.01[s]$ \\
\hline Mass of each link & $m_{1}=m_{2}=m_{3}=0.3[\mathrm{~kg}]$ \\
\hline Length of each link & $l_{1}=l_{2}=l_{3}=0.3[\mathrm{~m}]$ \\
\hline Distance between center & $l_{c l}=0.15[\mathrm{~m}]$ \\
\hline of Gravity and each joint & $l_{c 2}=l_{c 3}=0.15[\mathrm{~m}]$ \\
\hline Initial state & {$\left[\begin{array}{llllll}0 & 0 & 0 & 0 & 0 & 0\end{array}\right]$} \\
\hline Desired state & {$\left[\begin{array}{llllll}1 & 1 & 1 & 0 & 0 & 0\end{array}\right]$} \\
\hline
\end{tabular}
$d_{r}=10 \mathrm{~cm}, k_{\mathrm{i}}=0.3, k_{\mathrm{p}}=0.2$.

Tab.1 Setting parameters of simulations

Fig.5 and Fig.6 are the simulation results using virtual spring-damper method. Fig.5 shows the values of joint angles. The final joint angles are (1.042, 1.022, 1.025) $\mathrm{rad}$ which are very close to the desired value. Fig.6 is the motion diagram of underactuated system when there are obstacles and it is obviously that the linkages steer clear of the obstacle successfully.

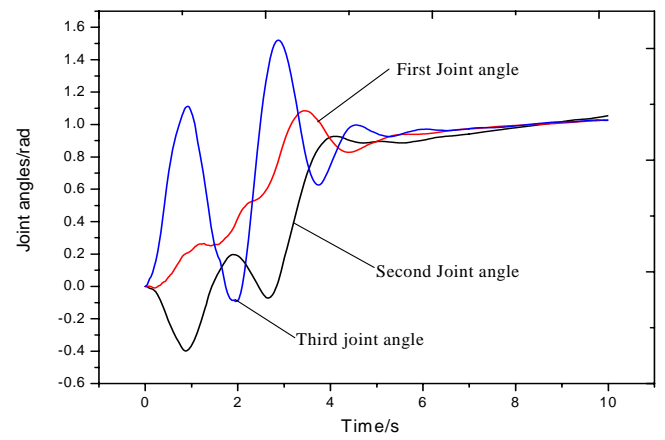

Fig.5 Time responses of joint angles using VSDM

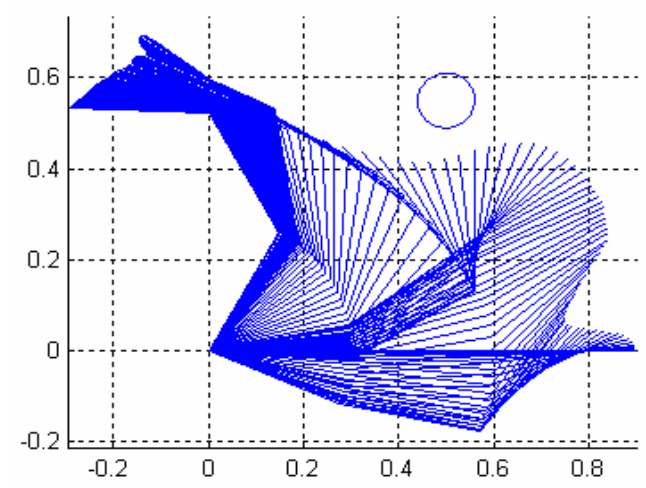

Fig.6 Rest-to-rest planning using VSDM: with obstacles in workspace 


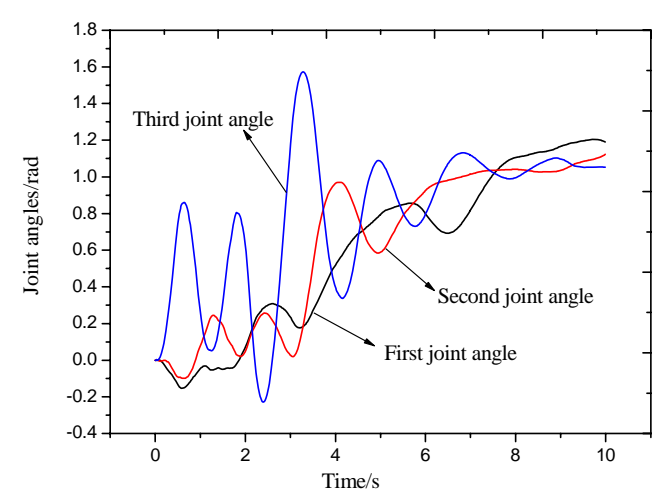

Fig.7 Time responses of joint angles using CTM

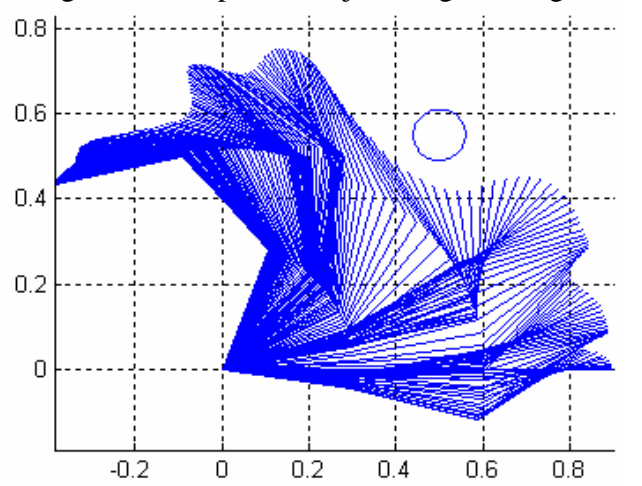

Fig.8 Rest-to-rest planning using CTM: with obstacles in workspace

Fig.7 and Fig.8 are the simulation results using computed torque method $^{15}$. The final joint angles are (1.191, 1.123, 1.052) rad. Fig.8 is the motion diagram of the underactuated robots. Although the linkages can steer clear of the obstacle, the relative errors of final joint angles are larger than that using VSDM, which means that the VSDM is more effective than CTM in collision-free trajectory planning of underactuated robot manipulators.

\section{Conclusion}

Dynamics analysis and obstacle avoidance of underactuated robot manipulators based on genetic algorithm is developed in this paper. This approach is based on representing the proximity of the arm to workspace obstacles by virtual force which is generated by the virtual spring-damper model. The partly stable controllers are derived and the goals are fulfilled by the switching of the partly stable controllers.

One of the major advantages of the proposed method is that the rigorous linearization or deformations of the original nonlinear system in the whole process are not considered. Because of the global optimization ability of genetic algorithm, it is equally applicable to model-based and sensor-based collision avoidance. Furthermore the proposed approach can be applied to both stationary and moving obstacles and also to multiple moving arms.

\section{Acknowledgements}

This work is supported by National Natural Science Foundation of China (Grant NO.50575002\&60705036), Beijing Natural Science Foundation (Grant NO.3062004), Beijing Education Committee Funding Project (Grant NO.KM200610005003) and PHR (IHLB).

\section{References}

1. Nakamura Y, Suzuki T, Koinuma N. Nonlinear behavior and control of a nonholonomic free-joint manipulator. IEEE Trans Robotics and Automation, Vol13 (6):853862(1997).

2. Spong $\mathrm{M}$ W. Underactuated Mechanical Systems. International Workshop on Control Problems in Robotics and Automation, San Diego, California, pages1-15(1997)

3. Bergerman M, XU Y, LU Y. Control of cooperative underactuated manipulators: a robust comparison study. Procs of 1st workshop on Robots and Mechatronics. Hong Kong, Vol.i, pp.279-286(1998)

4. Bergerman, M., Lee, C. and Xu, Y.S, "Dynamic Coupling of Underactuated Manipulators," Proc. of the IEEE Conference on Control Applications, pp.500505(1995).

5. Nakamura Y, Suzuki T, Koinuma N. Nonlinear behavior and control of a nonholonomic free-joint manipulator. IEEE Trans Robotics and Automation, Vol13 (6):853862(1997).

6. K.Kando. Motion planning with six degrees of freedom by multi strategic bidirectional heuristic free-space enumeration. IEEE Transaction of Robotics and Automation, Vol7,No.3,pp267-277(1991)

7. Hasegawa. T, Terasaki, H. Collision avoidance: divideand-conquer approach by space characterization and intermediate goals. IEEE Transaction on systems, man and cybernratics. Volume 18, Issue 3, May-June Page(s):337 - 347(1988)

8. Lozano P T. A simple motion planning algorithm for general robot manipulators. IEEE Journal of Robotics and Automation, RA-3 (3): 224-238(1987)

9. Kevin M. Lynch, Naoji Shiroma, Hirohiko Arai. Collision-Free Trajectory Planning for a 3-DOF Robot with a Passive Joint. The International Journal of Robotics Research, Vol. 19, No. 12, pp:1171-1184(2000)

10. Shingo Audo. A fast collision-free planning method for a general robot manipulator.Procedding of the 2003 IEEE International Conference on Robotics \& Automation.pp:2871-2877(2003) 
11. A. De Luca, R. Mattone, and G. Oriolo, "Steering a class of redundant mechanisms through end-effector generalized forces,” IEEE Trans.Robot. Automat.1998, vol. 14, no. 2, pp. 329-333

12. M. Reyhanoglu and A. J. van der Schaft, "Dynamics and control of second-order nonholonomic systems," in Benelux Meeting, Mierlo, The Netherlands, Mar (1996).

13. L. Udawatta, K. Watanabe, K. Izumi, K. Kiguchi. Control of underactuated robot manipulators using switching. Computed torque method: GA based approach. Soft Computing - A Fusion of Foundations, Methodologies and Applications.Volume 8, Number 1(2003).

14. Mitsuo Gen, Runwei Cheng. Genetic Algorithms and Engineering Optimization.ISBN:7-302-07482-Beijing: Tsing Hua University Press.2004, 1.

15. L.Udawatta, K.Watanabe, K.Izumi, K.Kiguchi .Obstacle Avoidance of Three-DOF Underactuated Manipulator by Using Switching Computed Torque Method, Trans. on Control, Autmation and Systems Engineering.Vol. 4, No. 4, pp. 347-355(2002). 